Scientific Visualization, 2020, volume 12, number 1, pages 103 - 111, DOI: 10.26583/sv.12.1.09

\title{
Modeling the development of Kelvin-Helmholtz instability in problems of high energy density physics
}

\author{
N.V. Zmitrenko',A, P.A. Kuchugov²,A, M.E. Ladonkina3,A, V.F. Tishkin4,A \\ Keldysh Institute of Applied Mathematics of Russian Academy of Sciences \\ ${ }^{1}$ ORCID: oooo-0003-0973-4388, zmitrenko@imamod.ru \\ 2 ORCID: oooo-0003-3240-2963, pkuchugov@gmail.com \\ 3 ORCID: 0000-0001-7596-1672, ladonkina@imamod.ru \\ 4 ORCID: 0000-0001-7295-7002, v.f.tishkin@mail.ru
}

\begin{abstract}
$\underline{\text { Abstract }}$
With the construction of powerful laser facilities, research in high energy density physics has received a new push of the development due to the possibility of making experiments. One of the main directions during many years remains the study of the development of initial perturbations under the various hydrodynamic instabilities. Due to the development of modern diagnostic methods in the last decade, these phenomena were experimentally observed under conditions of high energy densities, which made it possible to verify the available numerical codes and also to obtain new data.

The results of mathematical modeling of the development of Kelvin-Helmholtz instability in the conditions of irradiation of flat targets using an OMEGA laser facility are presented in this work. The vortex growth rate is compared with available experimental data. Taking into account the simplifications made in the numerical formulation of the problem, the various data are in satisfactory agreement with each other. The visualization of the flow made it possible to fully analyze its structure, determine the sizes of the characteristic vortices, and also reveal the differences between the $3 \mathrm{D}$ and $2 \mathrm{D}$ variants associated with the relaxation of the flow in transverse directions. The implementation of the visualization module is based on the use of VTK XML parallel format for storing data for analysis.
\end{abstract}

Keywords: Kelvin-Helmholtz instability, flow visualization, high energy density physics, mathematical modeling.

\section{Introduction}

As it is known, the conditions for the development of classical hydrodynamic instabilities, such as the Rayleigh-Taylor (RT), Richtmeyer-Meshkov (RM) and Kelvin-Helmholtz (KH) instabilities, are realized in a wide range of natural phenomena and anthropogenic systems. The scale of such phenomena and systems may differ by orders, for example, from a supernova explosion to compression of a capsule with thermonuclear fuel. Due to the laws of similarity, the structure of the flow, even in so many different tasks, remains similar. And for many decades, this has attracted the attention of researchers from various fields, such as plasma physics, geophysics, meteorology, astrophysics, and others, to the study of the perturbations growth dynamics under the mentioned instabilities.

Despite a long period of research on these problems, which can already be called classical, interest in them does not fade away. This is due, firstly, to the complexity of the phenomenon itself, which consists in the existence of various regimes development of perturbations. It is known that the initial spectrum of perturbations largely determines their further evolution and transition to turbulent mixing [1-5]. Secondly, the flow structure and mixing can signifi- 
cantly affect the conjugate physical processes that must be taken into account when studying problems such as laser fusion.

In further, namely this direction will be born in mind as one of the most relevant in modern plasma physics. For example, due to the growth of perturbations, the surface area increases through which the $\alpha$-particles formed as a result of the fusion of light nuclei $\mathrm{D}$ and $\mathrm{T}$ leave the central hot region of the thermonuclear target, which reduces the self-heating of the fuel. In addition, in the presence of disturbances, the amount of energy transferred to the central region by X-rays in indirect compression schemes changes. Additional preheating of the target prevents its good compression, which is necessary to obtain a high neutron yield. Turbulent mixing of different parts of the target can also have different consequences on the compression and burning efficiency of thermonuclear targets, introducing turbulent components into the flows of mass, momentum and energy. All this indicates the importance of studying the development of disturbances under extreme conditions when a substance is irradiated with powerful laser pulses from tens of kilojoules to several megajoules. The same conclusion is confirmed by regularly carried out experiments on the study of hydrodynamic instabilities at the largest operating laser systems OMEGA and NIF.

In this paper, it is considered the statement of the problem, which is devoted to the study of the dynamics perturbations growth due to KHI by numerical simulation.

\section{Experimental setup}

The first successful experiments to observe the development of KHI under conditions characteristic for the problems of high energy density physics were performed on OMEGA laser facility $[4,6]$. The essence of the experiment consisted in irradiating a flat composite target inside a beryllium shock tube with laser radiation with a total energy of the order of several tens of kilojoules. Between the upper and lower blocks of the target, single-mode initial perturbations were formed. The shock wave created due to evaporation under the action of a laser pulse from an ablator when passing through these blocks caused a shear flow due to different front velocities in various materials. As a result, the initial perturbations began to develop under the KHI. The obtained experimental data make it possible to verify both new physical models that allow one to describe the evolution of various perturbations, as well as newly created numerical methods and numerical codes.

\section{Problem formulation for modeling}

For numerical simulation, two-dimensional and three-dimensional formulations of the problem are considered. A schematic representation of the computational domain and its linear dimensions in microns are shown in Fig. 1.

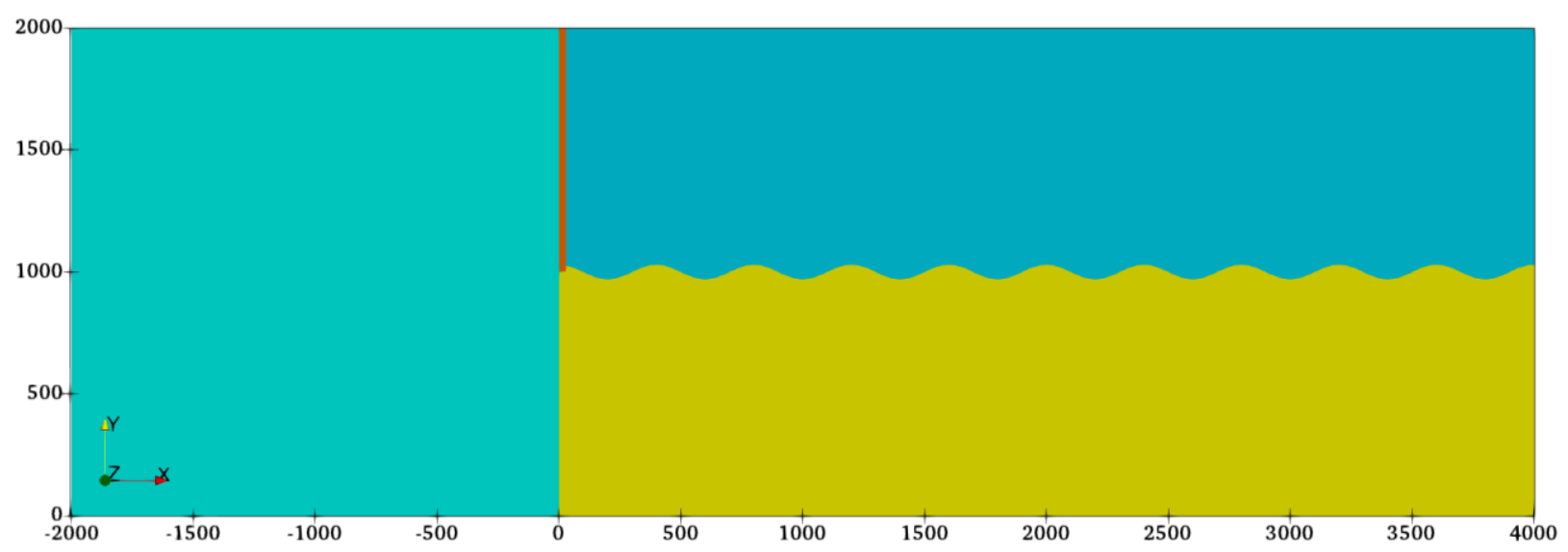

a) 


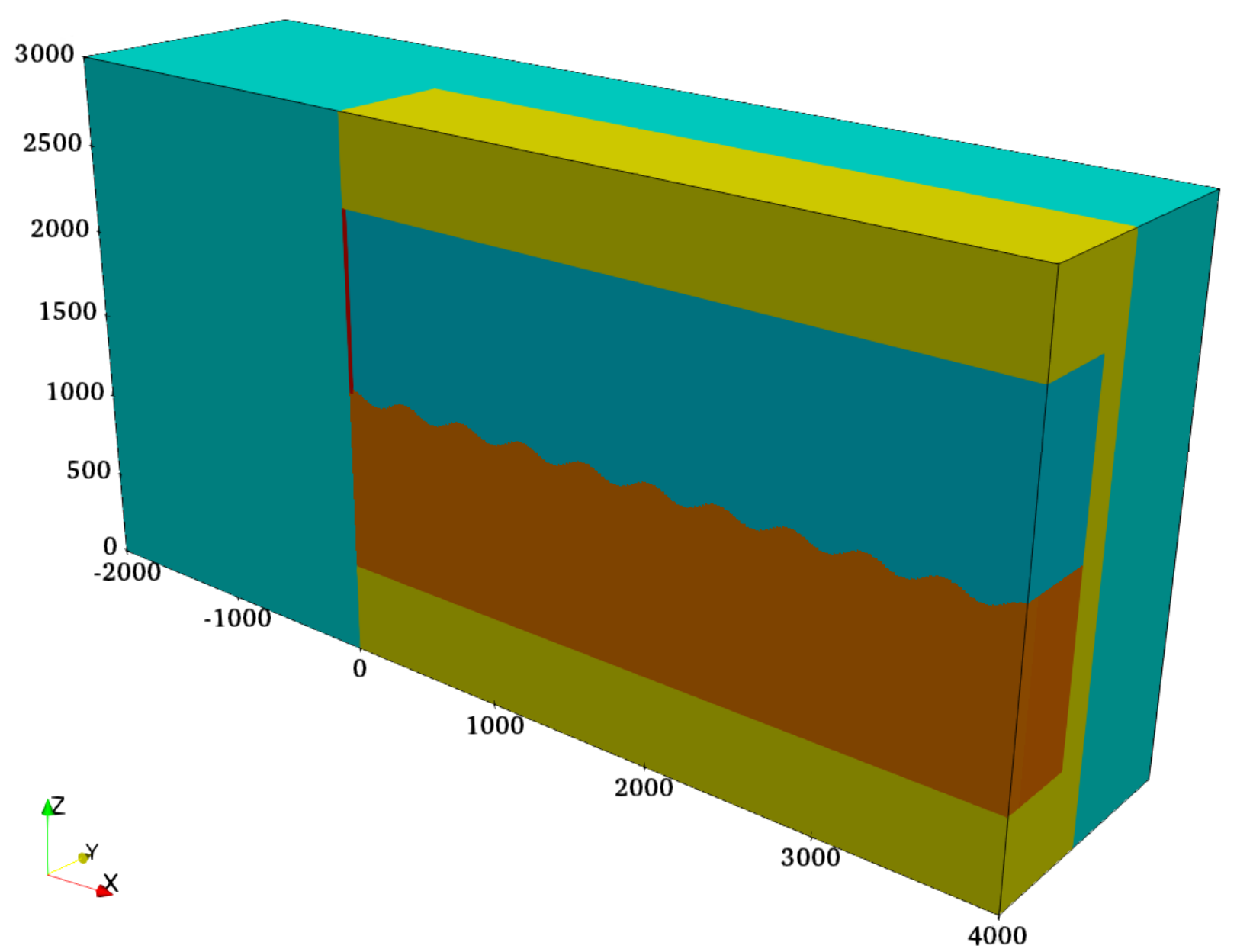

b)

Figure 1. Schematic representation of the computational domain and its main linear sizes in microns, as well as the numbers of subdomains: a) 2D setup, b) 3D setup.

The parameters of substances, such as density, specific heats ratio, specific heat capacity, and also temperature at the initial moment of time are given in Table 1.

Table 1. The initial density and temperature of various subdomains, as well as the specific heats ratio and specific heat capacity at constant volume.

\begin{tabular}{|l|l|l|l|l|}
\hline Nos. & $\begin{array}{l}\text { Density, } \\
\mathrm{g} / \mathrm{cm}^{3} \\
(2 \mathrm{D} / 3 \mathrm{D})\end{array}$ & $\begin{array}{l}\text { Specific } \\
\text { heats ratio }\end{array}$ & $\begin{array}{l}\text { Specific heat capacity at con- } \\
\text { stant } \\
10^{4} \mathrm{~kJ} / \mathrm{g} / \mathrm{keV}\end{array}$ & $\begin{array}{l}\text { Temperature, keV } \\
(2 \mathrm{D} / 3 \mathrm{D})\end{array}$ \\
\hline 1 & 0.01 & $5 / 3$ & 3.6 & 0.01 \\
\hline 2 & 1.05 & $5 / 3$ & 10 & $3.357 \cdot 10^{-5} / 6.905 \cdot 10^{-4}$ \\
\hline 3 & 0.1 & $7 / 5$ & 10 & $5.875 \cdot 10^{-4} / 0.01208$ \\
\hline 4 & $1.41 / 1.45$ & $5 / 3$ & 10 & $2.5 \cdot 10^{-5} / 5 \cdot 10^{-4}$ \\
\hline 5 & 1.42 & $5 / 3$ & 10 & $-/ 5 \cdot 106 \cdot 10^{-4}$ \\
\hline 6 & 1.84 & $5 / 3$ & 7.174 & $-/ 5.106 \cdot 10^{-4}$ \\
\hline
\end{tabular}

In a two-dimensional formulation, substance No. 2 occupies a subdomain $30 \mu \mathrm{m}$ wide $(0<x<30 \mu \mathrm{m})$ and a height of $1000 \mu \mathrm{m} 1000<y<2000 \mu \mathrm{m})$, subdomain $0<x<4000$ $\mu \mathrm{m}$ and $0<y<1000 \mu \mathrm{m}$ takes substance No. 4, and above - substance No. 3 . 
In the three-dimensional setup, the dimensions along $x$ and $z$ for substances 2-4 repeat the two-dimensional setup, the dimensions along $y$ are $0<y<500 \mu \mathrm{m}$ for substances No. 2 and 3, $0<y<100 \mu \mathrm{m}$ for No. 4, subregion $100<y<500 \mu \mathrm{m}$ is occupied by substance No. 5 . Subregions 2-5 are located inside the shell of substance No. 6 with $y$ from o to $700 \mu \mathrm{m}$. Substance No. 1 is external to $2-6$ ones.

Single-mode perturbations between subregions No. 3 and No. 4 have a wavelength of $400 \mu \mathrm{m}$ and an amplitude of $30 \mu \mathrm{m}$.

The absorption of laser radiation is simulated by a local mass-uniform energy release in subregion No. 2. The time dependence of the source $f(t)=\frac{1}{\varepsilon_{0}} \frac{d \varepsilon}{d t}$, where $\varepsilon_{0}=35 \mathrm{~kJ} / \mathrm{g}$, has the form of an isosceles trapeze, bases difference of which is $0.4 \mathrm{~ns}$.

A Cartesian cubic grid with a step of $2 \mu \mathrm{m}$ for two-dimensional formulation and $10 \mu \mathrm{m}$ for three-dimensional one is used for discrete representation of computational domain.

\section{Description of numerical method and program imple- mentation}

Modeling of the problems was carried out using the NUT3D software package [1-3]. The NUT3D numerical code solves the multicomponent gas dynamics equations: the equations for the various components (partial densities $\rho C_{\alpha}$, where $C_{\alpha}$ is the mass concentration) are solved together with the Euler equations. The difference algorithm is based on a TVD secondorder spatial accuracy scheme with limiters of anti-diffusion fluxes [1]. The fluxes at the cell boundaries in this paper are calculated using analytical expressions for solving the Riemann problem for ideal gas, which is a multi-component medium with possibly different specific heats ratios for different components. Integration over time is performed using the predictorcorrector method, which allows us to achieve a second order of accuracy. The time step value is recalculated during the calculation in accordance with the Courant-Friedrichs-Lewy condition.

Parallel implementation is based on the method of domain decomposition. The boundary conditions and inter-processes exchanges are implemented using method of ghost cells. It is also possible to involve GPUs available on computational node for calculations [7].

Recovery points containing the calculation results are stored in a binary file of a certain format, and each MPI-process writes its own file. For visualization of simulation results, VTK XML [8] parallel format and ParaView program are used. When generating output files for visualization, data compression is additionally used to reduce the size of the occupied disk space. It should be noted that one or another way of storing recovery points and visualization files can have a critical effect on the total calculation time.

\section{Numerical results}

Using the NUT3D software package described above, the simulation was performed according to the two discussed problem setups up to a time moment of $80 \mathrm{~ns}$, corresponding to the arrival of the shock wave to the right edge of the computational domain. In Fig. 2 and 3 the corresponding evolution of the density distribution is shown. 


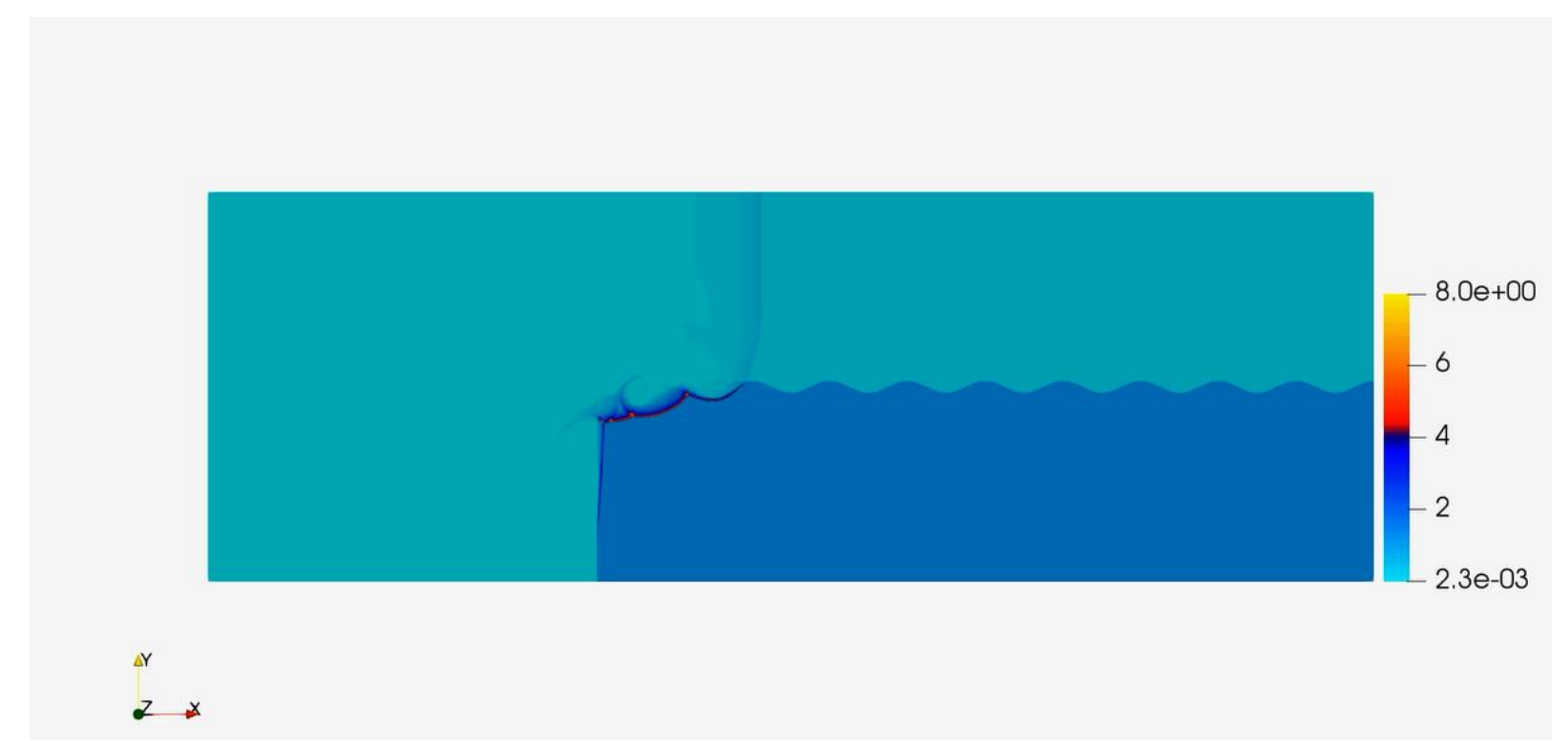

Figure 2. Evolution of the density distribution in the case of a two-dimensional problem setup.

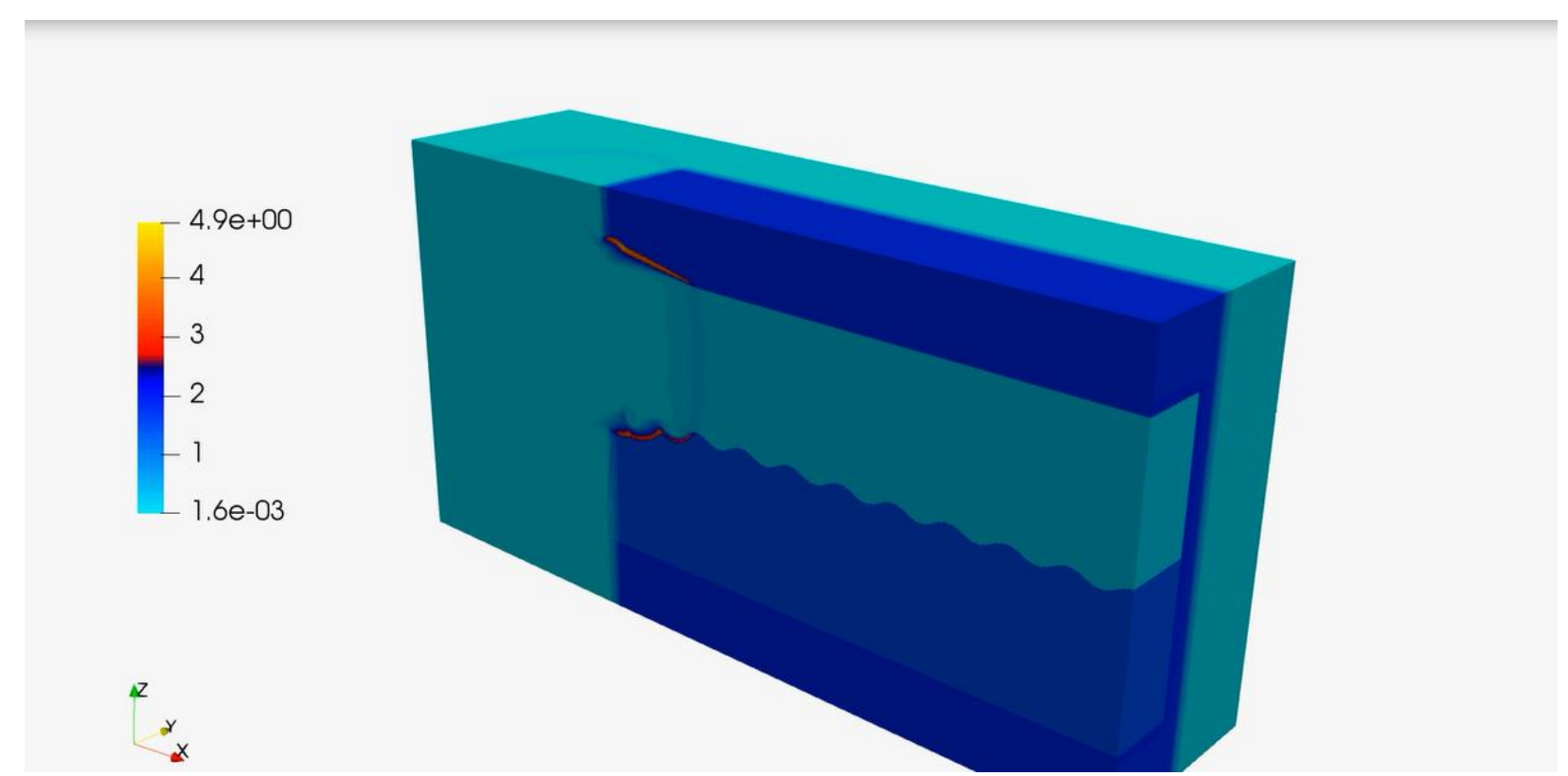

Figure 3. Evolution of the density distribution in the case of a three-dimensional problem setup.

During the passage of the shock wave along the perturbed surface, the formation of vortex structures characteristic of KHI is observed. In the region behind the main shock wave, a complex shock-wave flow pattern is observed due to the multiple reflection of secondary shock waves from different interfaces. In the three-dimensional case, in contrast to the twodimensional case, flow relaxation in the transverse direction is observed (see Fig. 4). 


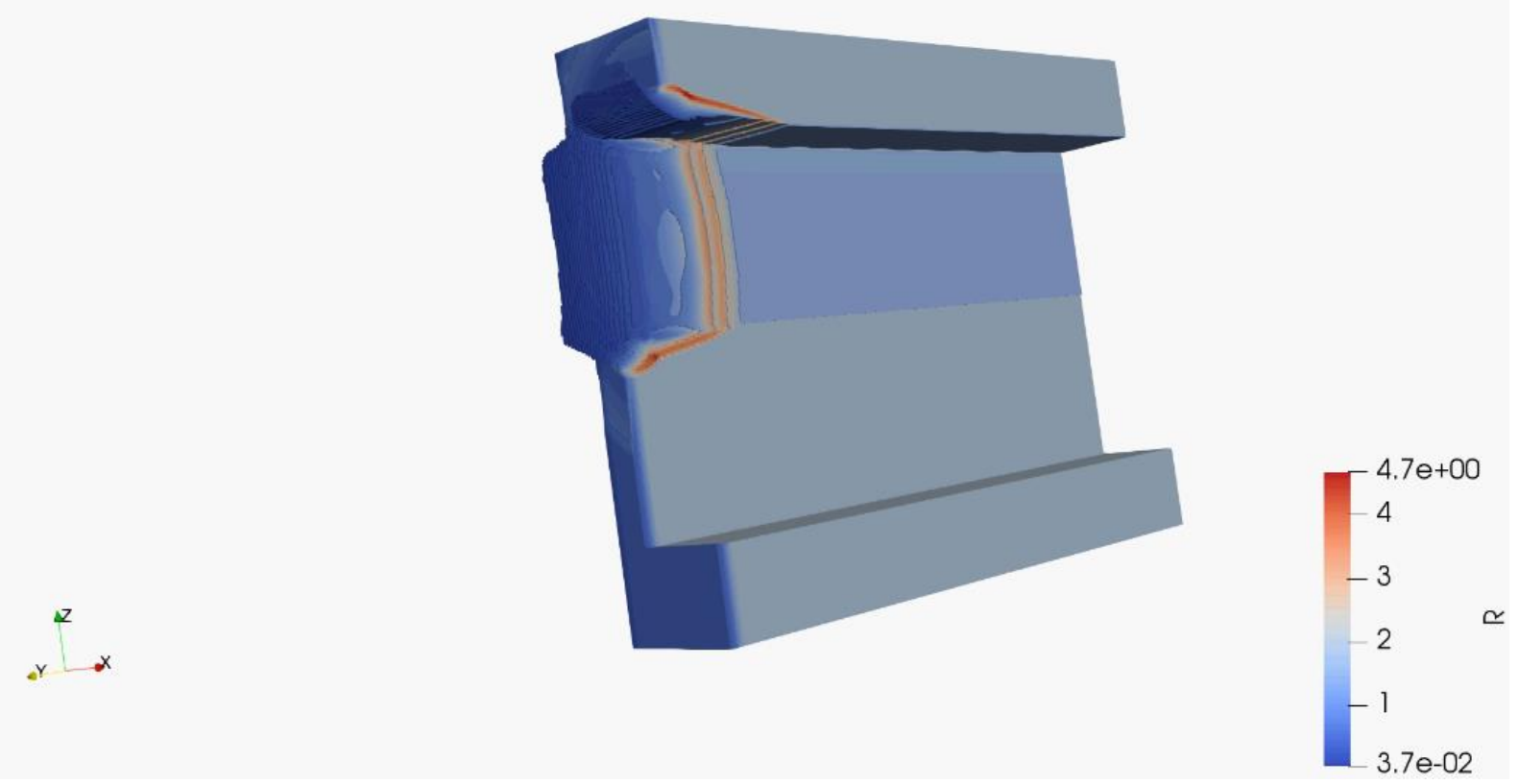

Figure 4. Evolution of the shape of a region with substance No. 6 in the case of a threedimensional problem setup.

Bubbles growth in this case extends to areas of neighboring substances, namely, No. 5 and 6 . For a quantitative comparison of the results, we use the data from [9] and compare the growth dynamics of the height of the vortices characteristic of the KHI. The relevant data are shown in Fig. 5.

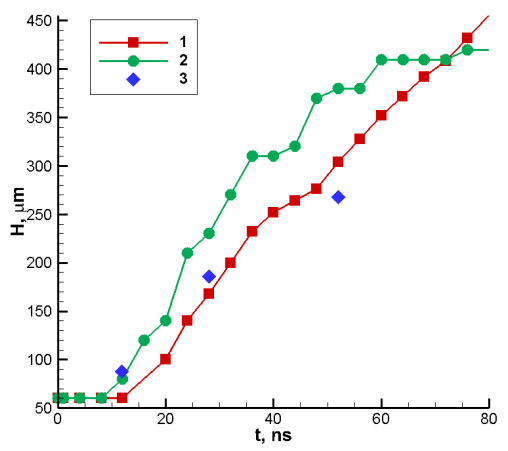

a)

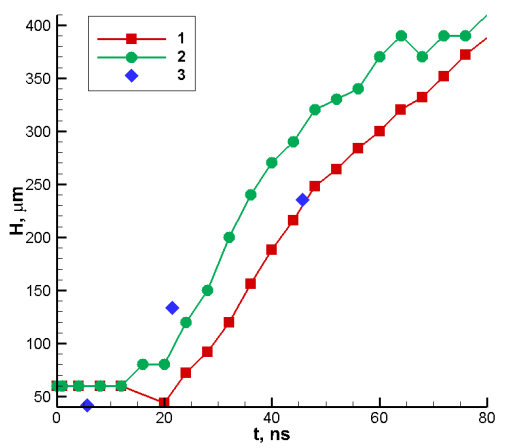

b)

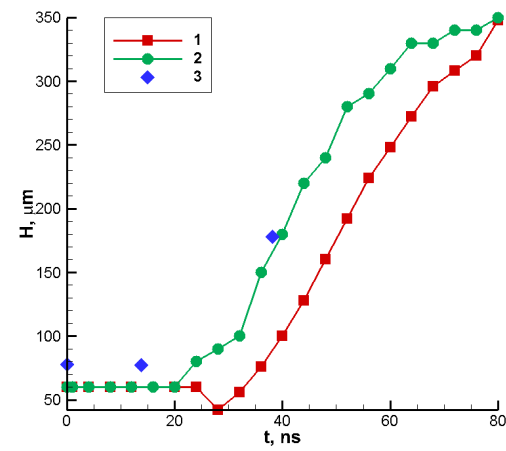

c)

Figure 5. Dependence of the height a) of the 2nd, b) of the 3 rd and c) of the 4 th vortex on time: 1 - 2D, 2 - 3D, 3 - experimental data.

These figures show, firstly, that despite the simplified formulation of the problem for the numerical simulation, the vortex amplitudes, obtained on the basis of experimental data, are reproduced quite well in numerical calculations. Secondly, it should be noted that the increase in the amplitude of the perturbations is close to linear with a speed of $a=7-8 \mathrm{~km} / \mathrm{s}$ in certain ranges $(H=a t)$. Thirdly, it is seen that the amplitudes of the vortices in the threedimensional case are higher than in the two-dimensional one. This fact is consistent with a linear analysis of the increments of KHI [10], which gives a higher growth rate of perturbations in $3 \mathrm{D}$ geometry as compared to $2 \mathrm{D}$ at equal wavelengths at the initial time.

\section{Conclusion}

In this work we analyze in detail an experimental data obtained at the OMEGA facility, aimed at studying the development of Kelvin-Helmholtz instability under conditions of intense (of the order of $10^{14} \mathrm{~W} / \mathrm{cm}^{2}$ ) laser irradiation of the target. The work describes a numerical code 
(NUT), with the help of which an comprehensive modeling of the processes under study was performed. The experimental data are in good agreement with the calculated ones. This indicates, first of all, in favor of the fact that the selected physical model (gas dynamics of an ideal gas) turns out to be a suitable tool for studying such laser experiments characterized by significant material temperatures (of the order of $100 \mathrm{eV}$ ) and densities (of the order of solid and gas densities). The results obtained can serve as the basis for the necessary estimates of the development of hydrodynamic instabilities in other experiments and the development of models for the theoretical description of turbulence and mixing processes.

The developed numerical code NUT3D used here to simulate the described problems (both in $2 \mathrm{D}$ and in $3 \mathrm{D}$ versions) is a practically useful tool for studying the problems of generation and development of hydrodynamic instabilities. The implemented module for visualizing flows allows you to analyze their structure in detail, as well as perform data processing in order to obtain quantitative integral characteristics.

\section{Acknowledgements}

The results of the work were obtained using computational resources of MCC NRC «Kurchatov Institute», http://computing.nrcki.ru/ as well as the equipment of the shared research facilities of HPC computing resources at Lomonosov Moscow State University.

\section{References}

1. Tishkin V.F., Nikishin V.V., Popov I.V., Favorski A.P. Raznostnye skhemy trekhmernoj gazovoj dinamiki dlya zadachi o razvitii neustojchivosti Rihtmajera-Meshkova [Finite difference schemes of three-dimensional gas dynamics for the study of RichtmyerMeshkov instability] // Matem. Mod., 7, 5, 1995, pp. 15-25 [in Russian].

2. Kuchugov P.A. Dinamika processov turbulentnogo peremeshivaniya $\mathrm{v}$ lazernyh mishenyah [Dynamics of turbulent mixing in laser fusion targets] // PhD Thesis, Keldysh Institute of Applied Mathematics of RAS, 2014 [in Russian].

3. Ladonkina M.E. CHislennoe modelirovanie turbulentnogo peremeshivaniya $\mathrm{S}$ ispol'zovaniem vysokoproizvoditel'nyh sistem [Numerical modeling of turbulent mixing using high-performance computers] // PhD Thesis, Institue of Mathematical Modeling of RAS, 2005 [in Russian].

4. Harding E.C., Hansen J.F., Hurricane O.A., Drake R.P., Robey H.F., Kuranz C.C., Remington B.A., Bono M.J., Grosskopf M.J., and Gillespie R.S. Observation of a KelvinHelmholtz Instability in a High-Energy-Density Plasma on the Omega Laser // Phys. Rev. Lett., 103, 045005, 2009 (doi: 10.1103/PhysRevLett.103.045005).

5. Kuchugov P., ZmitrenkoN., RozanovV., YanilkinYu., Sin'kovaO., StatsenkoV., Chernyshova O., The Evolution Model of the Rayleigh-Taylor Instability Development // Journal of Russian Laser Research, 33, 6, 517-530, 2012 (doi: 10.1007/s10946-012-9310y).

6. Hurricane O.A., Hansen J.F., Robey H.F., Remington B.A., Bono M.J., Harding E.C., Drake R.P., Kuranz C.C. A high energy density shock driven Kelvin-Helmholtz shear layer experiment // Physics of Plasmas, 16, 056305, 2009 (doi: 10.1063/1.3096790).

7. Kuchugov P.A. Organizing of Intra-node CPU-GPUs Communications in Multi-GPU Numerical Code for Modeling Laser Fusion Problems // Proceedings of International scientific conference "Parallel Computational Technologies (PCT'2018)", 2-6 april 2018, Rostov-on-Don, Russia,2018, P. 126-138.

8. The VTK User's Guide. File Formats for VTK Version 4.2, https://vtk.org/Wiki/VTK XML Formats.

9. Raman K.S., Hurricane O.A., Park H.-S., Remington B.A., Robey H., Smalyuk V.A., Drake R.P., Krauland C.M., Kuranz C.C., Hansen J.F., Harding E.C. Three-dimensional modeling and analysis of a high energy density Kelvin-Helmholtz experiment // Physics of Plasmas, 19, 092112, 2012 (doi: 10.1063/1.4752018). 
10. Zmitrenko N.V., Rozanov V.B. Inkrementy neustojchivosti Kel'vina-Gel'mgol'ca v zadachah lazernogo termoyadernogo sinteza [Growth rates of Kelvin-Helmholtz instability in laser fusion problems] // Preprint of Lebedev Physical Institute of RAS No. 16, 1992 [in Russian]. 